\title{
BOSONIC FORMULAS FOR AFFINE BRANCHING FUNCTIONS
}

\author{
E.FEIGIN
}

\begin{abstract}
In this paper we derive two bosonic (alternating sign) formulas for branching functions for general affine Kac-Moody Lie algebra $\mathfrak{g}$. Both formulas are given in terms of Weyl group and string functions of $\mathfrak{g}$.
\end{abstract}

\section{INTRODUCTION}

Let $\mathfrak{g}$ be an affine Kac-Moody Lie algebra, $\mathfrak{g}_{f i n}$ be the corresponding simple finite-dimensional algebra,

$$
\mathfrak{g}=\mathfrak{g}_{\text {fin }} \otimes \mathbb{C}\left[t, t^{-1}\right] \oplus \mathbb{C} K \oplus \mathbb{C} d,
$$

where $K$ is a central element and $\left[d, x \otimes t^{i}\right]=-i x \otimes t^{i}$ for $x \in \mathfrak{g}$. We fix the Cartan decomposition $\mathfrak{g}=\mathfrak{n} \oplus \mathfrak{h} \oplus \mathfrak{n}_{-}$, with

$$
\mathfrak{h}=\mathfrak{h}_{\text {fin }} \oplus \mathbb{C} K \oplus \mathbb{C} d,
$$

where $\mathfrak{h}_{\text {fin }}$ is the Cartan subalgebra of $\mathfrak{g}_{\text {fin }}$. We also denote

$$
\mathfrak{g}^{\prime}=[\mathfrak{g}, \mathfrak{g}]=\mathfrak{g} \otimes \mathbb{C}\left[t, t^{-1}\right] \oplus \mathbb{C} K, \quad \mathfrak{h}^{\prime}=\mathfrak{h}_{\text {fin }} \oplus \mathbb{C} K .
$$

Let $P_{k}^{+} \hookrightarrow \mathfrak{h}^{*}$ be the set of all dominant integrable level $k$ weights of $\mathfrak{g}$. We denote by $P_{k}^{\prime+} \hookrightarrow \mathfrak{h}^{\prime *}$ the image of $P_{k}^{+}$with respect to the restriction map $\mathfrak{h}^{*} \rightarrow \mathfrak{h}^{\prime *}, \lambda \mapsto \lambda^{\prime}$. For $\lambda \in P_{k}^{+}$we denote by $L_{\lambda}$ the corresponding irreducible highest weight $\mathfrak{g}$ module and by $L_{\lambda^{\prime}}$ a $\mathfrak{g}^{\prime}$ module which coincides with $L_{\lambda}$ as a vector space and the action of $\mathfrak{g}^{\prime}$ is a restriction of the action of $\mathfrak{g}$.

For $\lambda_{1} \in P_{k_{1}}^{\prime}, \lambda_{2} \in P_{k_{2}}^{\prime+}$ consider a decomposition of the tensor product of $\mathfrak{g}^{\prime}$-modules

$$
L_{\lambda_{1}^{\prime}} \otimes L_{\lambda_{2}^{\prime}}=\bigoplus_{\mu^{\prime} \in P_{k_{1}+k_{2}}^{\prime+}} L_{\mu^{\prime}} \otimes C_{\lambda_{1}^{\prime} \lambda_{2}^{\prime}}^{\mu^{\prime}}
$$

Note that $C_{\lambda_{1}^{\prime} \lambda_{2}^{\prime}}^{\mu^{\prime}}$ can be considered as a space of highest weight vectors of $\mathfrak{h}^{\prime}$-weight $\mu^{\prime}$ in $L_{\lambda_{1}^{\prime}} \otimes L_{\lambda_{2}^{\prime}}$. To define a character of $C_{\lambda_{1}^{\prime} \lambda_{2}^{\prime}}^{\mu^{\prime}}$ we assume $\lambda_{1}(d)=\lambda_{2}(d)=\mu(d)=0$ (note that for any $\lambda^{\prime} \in P_{k}^{\prime+}$ there exists $\bar{\lambda} \in P_{k}^{+}$ 
such that $\bar{\lambda}(d)=0$ and $\left.\left.\bar{\lambda}\right|_{\mathfrak{h}^{\prime}}=\lambda^{\prime}\right)$. Then we obtain a grading by the operator $d$ on $L_{\lambda_{1}^{\prime}} \otimes L_{\lambda_{2}^{\prime}}$. Set

$$
c_{\lambda_{1}^{\prime} \lambda_{2}^{\prime}}^{\mu^{\prime}}(q)=\operatorname{ch}_{q} C_{\lambda_{1}^{\prime} \lambda_{2}^{\prime}}^{\mu^{\prime}}=\left.\operatorname{Tr} q^{d}\right|_{C_{\lambda_{1}^{\prime} \lambda_{2}^{\prime}}^{\mu^{\prime}}} .
$$

These functions are called $\mathfrak{g}$ branching functions. We note that in the conformal field theory branching functions appear as characters of spaces of states of coset theories. These characters differs from $c_{\lambda_{1}^{\prime} \lambda_{2}^{\prime}}^{\mu^{\prime}}(q)$ by an extra factor $q^{\triangle_{\lambda_{1}^{\prime}}+\triangle_{\lambda_{2}^{\prime}}-\triangle_{\mu^{\prime}}}$, where $\triangle_{\lambda^{\prime}}$ is a conformal weight of $\lambda^{\prime}$ (see [DMS]).

There exist different approaches to the study of $c_{\lambda_{1}^{\prime} \lambda_{2}^{\prime}}^{\mu^{\prime}}(q)$ (see for example [BNY, KMQ, [R, DJKMO, [S1, S2, SS, [F, [FOW]). These approaches give different types formulas for some particular cases of branching functions. In our paper we use homological technique to derive two bosonic formulas for $c_{\lambda_{1}^{\prime} \lambda_{2}^{\prime}}^{\mu^{\prime}}(q)$ for general affine Kac-Moody algebras (note that similar approach is utilized in FFJMT, $[\mathrm{FF}, \mathrm{F}$ ). Let us briefly describe our results. Recall the Garland-Lepowsky theorem:

$$
H_{p}\left(\mathfrak{n}_{-}, L_{\mu}\right) \simeq \bigoplus_{\substack{w \in W \\ l(w)=p}} \mathbb{C}_{w * \mu},
$$

where $W$ is a Weyl group of $\mathfrak{g}, w * \mu$ is a shifted action of $\mathrm{W}$ and $l(w)$ is the length of $w$. Note that (2) is an isomorphism of $\mathfrak{h}$-modules and $\mathbb{C}_{w * \mu}$ is one-dimensional $\mathfrak{h}$-module of the weight $w * \mu$. From (2) we obtain that for $\mu, \nu \in P_{k}^{+}$homology $H_{p}\left(\mathfrak{n}_{-}, L_{\mu}\right)^{\nu}$ (superscript denotes the corresponding $\mathfrak{h}$-weight subspace) vanishes if $p>0$ or $\mu \neq \nu$. In addition

$$
H_{0}\left(\mathfrak{n}_{-}, L_{\mu}\right)^{\mu} \simeq \mathbb{C}_{\mu} .
$$

Therefore from (11) we obtain

$$
H_{p}\left(\mathfrak{n}_{-}, L_{\lambda_{1}} \otimes L_{\lambda_{2}}\right)^{\mu^{\prime}} \simeq C_{\lambda_{1}^{\prime} \lambda_{2}^{\prime}}^{\mu^{\prime}} \delta_{0, p}
$$

and so

$$
\sum_{p \geq 0}(-1)^{p} \operatorname{ch}_{q} H_{p}\left(\mathfrak{n}_{-}, L_{\lambda_{1}} \otimes L_{\lambda_{2}}\right)^{\mu^{\prime}}=c_{\lambda_{1}^{\prime} \lambda_{2}^{\prime}}^{\mu^{\prime}}(q) .
$$

We now compute the same Euler characteristics using the BGG-resolution of $L_{\lambda_{1}}$ :

$$
\ldots \rightarrow F_{p} \rightarrow \ldots \rightarrow F_{0} \rightarrow L_{\lambda_{1}} \rightarrow 0
$$

where $F_{p}=\bigoplus_{l(w)=p} M_{w * \lambda_{1}}$ and $M_{w * \lambda_{1}}$ is the corresponding Verma module. Tensoring the BGG-resolution by $L_{\lambda_{2}}$ we obtain the $\mathrm{U}\left(\mathfrak{n}_{-}\right)$-free resolution of $L_{\lambda_{1}} \otimes L_{\lambda_{2}}$ :

$$
\ldots \rightarrow F_{p} \otimes L_{\lambda_{2}} \rightarrow \ldots \rightarrow F_{0} \otimes L_{\lambda_{2}} \rightarrow L_{\lambda_{1}} \otimes L_{\lambda_{2}} \rightarrow 0 .
$$


Then the homology $H_{p}\left(\mathfrak{n}_{-}, L_{\lambda_{1}} \otimes L_{\lambda_{2}}\right)^{\mu^{\prime}}$ can be counted as homology of a complex

(6) $\ldots \rightarrow\left[\mathbb{C} \otimes_{\mathrm{U}\left(\mathfrak{n}_{-}\right)}\left(F_{p} \otimes L_{\lambda_{2}}\right)\right]^{\mu^{\prime}} \rightarrow \ldots \rightarrow\left[\mathbb{C} \otimes_{\mathrm{U}\left(\mathfrak{n}_{-}\right)}\left(F_{0} \otimes L_{\lambda_{2}}\right)\right]^{\mu^{\prime}} \rightarrow 0$.

Therefore the Euler characteristics (3) is given by the formula

$$
\sum_{p \geq 0}(-1)^{p} \sum_{l(w)=p} q^{\left(w * \lambda_{1}\right) d} \operatorname{ch}_{q}\left(L_{\lambda_{2}}\right)^{\left(\mu-w * \lambda_{1}\right)^{\prime}} .
$$

We thus obtain our first bosonic formula for $c_{\lambda_{1}^{\prime} \lambda_{2}^{\prime}}^{\mu^{\prime}}(q)$.

To get the second formula we replace the "product" $L_{\lambda_{1}} \otimes L_{\lambda_{2}}$ by the "fraction" $L_{\mu} \otimes L_{\lambda_{1}}^{*}$ and consider the homology $H_{p}\left(\mathfrak{n}_{-}, L_{\mu} \otimes L_{\lambda_{1}}^{*}\right), \lambda_{1} \in P_{k_{1}}^{+}$, $\mu \in P_{k_{1}+k_{2}}^{+}$. We prove that

$$
H_{p}\left(\mathfrak{n}_{-}, L_{\mu} \otimes L_{\lambda_{1}}^{*}\right)^{\lambda_{2}^{\prime}}=0 \text { for } p>0
$$

and

$$
H_{0}\left(\mathfrak{n}_{-}, L_{\mu} \otimes L_{\lambda_{1}}^{*}\right)^{\lambda_{2}^{\prime}} \simeq\left(C_{\lambda_{1}^{\prime} \lambda_{2}^{\prime}}^{\mu^{\prime}}\right)^{*}
$$

We thus obtain that

$$
\sum_{p \geq 0}(-1)^{p} \operatorname{ch}_{q} H_{p}\left(\mathfrak{n}_{-}, L_{\mu} \otimes L_{\lambda_{1}}^{*}\right)^{\lambda_{2}^{\prime}}=c_{\lambda_{1}^{\prime} \lambda_{2}^{\prime}}^{\mu^{\prime}}\left(q^{-1}\right) .
$$

Using the BGG-resolution of $L_{\mu}$ we again rewrite this Euler characteristics in terms of the characters of spaces $\left(\mathbb{C} \otimes_{\mathrm{U}\left(\mathfrak{n}_{-}\right)}\left(M_{w * \mu} \otimes L_{\lambda_{1}}^{*}\right)\right)^{\lambda_{2}^{\prime}}$. This gives the following formula:

$$
c_{\lambda_{1}^{\prime} \lambda_{2}^{\prime}}^{\mu^{\prime}}(q)=\sum_{p \geq 0}(-1)^{p} \sum_{l(w)=p} q^{-(w * \mu)(d)} \operatorname{ch}_{q}\left(L_{\lambda_{1}}\right)^{\left(w * \mu-\lambda_{2}\right)^{\prime}}
$$

(recall that we assume $\lambda_{1}(d)=\lambda_{2}(d)=\mu(d)=0$ ). The specialization of this formula to the simplest case $\mathfrak{g}=\widehat{\mathfrak{s l}}_{2}$ gives the formula from BNY, KMQ, R] in the form of $\mathbf{F}$. We note that (8) looks like (7), but the proof is much more complicated.

Our paper is organized as follows.

In Section 1 we fix affine Kac-Moody Lie algebras notations.

In Section 2 we derive our first formula for branching functions $c_{\lambda_{1}^{\prime} \lambda_{2}^{\prime}}^{\mu^{\prime}}(q)$ using the homology $H_{p}\left(\mathfrak{n}_{-}, L_{\lambda_{1}} \otimes L_{\lambda_{2}}\right)^{\mu^{\prime}}$.

In Section 3 we derive our second formula for $c_{\lambda_{1}^{\prime} \lambda_{2}^{\prime}}^{\mu^{\prime}}(q)$ using the homology of the "fraction" $H_{p}\left(\mathfrak{n}_{-}, L_{\mu} \otimes L_{\lambda_{1}}^{*}\right)^{\lambda_{2}^{\prime}}$.

In Section 4 we specialize formulas from Sections 2 and 3 to the simplest case $\mathfrak{g}=\widehat{\mathfrak{s l}_{2}}$.

Acknowledgements. This work was partially supported by RFBR Grant 06-01-00037 and LSS 4401.2006.2. 


\section{Affine KaC-Moody Lie Algebras}

In this section we fix our notations on the affine Kac-Moody Lie algebras. The main references are $\mathrm{Kac}$, Kum.

Let $\mathfrak{g}_{\text {fin }}$ be a simple finite-dimensional Lie algebra with the Cartan decomposition $\mathfrak{g}_{\text {fin }}=\mathfrak{n}_{\text {fin }} \oplus \mathfrak{h}_{\text {fin }} \oplus\left(\mathfrak{n}_{-}\right)_{\text {fin }}$.

Consider the corresponding affine algebra

$$
\mathfrak{g}=\mathfrak{g}_{\text {fin }} \otimes \mathbb{C}\left[t, t^{-1}\right] \oplus \mathbb{C} K \oplus \mathbb{C} d,
$$

where $K$ is a central element and $\left[d, x \otimes t^{i}\right]=-i x \otimes t^{i}$.

We fix the Cartan decomposition $\mathfrak{g}=\mathfrak{n} \oplus \mathfrak{h} \oplus \mathfrak{n}_{-}$, where

$$
\begin{gathered}
\mathfrak{n}=\mathfrak{n}_{\text {fin }} \otimes 1 \oplus \mathfrak{g}_{\text {fin }} \otimes t \mathbb{C}[t], \\
\mathfrak{h}=\mathfrak{h}_{\text {fin }} \oplus \mathbb{C} K \oplus \mathbb{C} d, \\
\mathfrak{n}_{-}=\left(\mathfrak{n}_{-}\right)_{\text {fin }} \otimes 1 \oplus \mathfrak{g}_{\text {fin }} \otimes t^{-1} \mathbb{C}\left[t^{-1}\right]
\end{gathered}
$$

and denote $\mathfrak{g}^{\prime}=[\mathfrak{g}, \mathfrak{g}]=\mathfrak{g}_{\text {fin }} \otimes \mathbb{C}\left[t, t^{-1}\right] \oplus \mathbb{C} K, \mathfrak{h}^{\prime}=\mathfrak{h}_{\text {fin }} \oplus \mathbb{C} K \hookrightarrow \mathfrak{g}^{\prime}$

Let $\alpha_{i}^{\vee} \in \mathfrak{h}, \alpha_{i} \in \mathfrak{h}^{*}, i=1, \ldots, n$, be simple coroots and roots. Note that $\alpha_{i}^{\vee}$ form a basis of $\mathfrak{h}_{\text {fin }} \oplus \mathbb{C} K$. We denote by $\mathfrak{s l}_{2}^{(i)}$ the $\mathfrak{s l}_{2}$ Lie algebra spanned by $e_{i}, \alpha_{i}^{\vee}, f_{i}$, where $e_{i}, f_{i}, i=1, \ldots, n$ are the Chevalley generators, $e_{i} \in \mathfrak{n}, f_{i} \in \mathfrak{n}_{-}$. We note that

$$
\mathfrak{n}=\bigoplus_{\alpha \in \triangle_{+}} \mathfrak{g}_{\alpha}, \mathfrak{n}_{-}=\bigoplus_{\alpha \in \triangle_{-}} \mathfrak{g}_{\alpha}
$$

where $\triangle_{+}$and $\triangle_{-}$are the sets of positive and negative roots and $\mathfrak{g}_{\alpha}=\{x \in$ $\mathfrak{g}:[h, x]=\alpha(h) x \forall h \in \mathfrak{h}\}$. Spaces $\mathfrak{g}_{\alpha_{i}}$ and $\mathfrak{g}_{-\alpha_{i}}$ are spanned by $e_{i}$ and $f_{i}$. Let

$$
\mathfrak{u}_{-}^{(i)}=\bigoplus_{\substack{\alpha \in \triangle_{-} \\ \alpha \neq-\alpha_{i}}} \mathfrak{g}_{\alpha}
$$

Note that

$$
\mathfrak{g}_{-\alpha_{i}} \simeq \mathfrak{n}_{-} / \mathfrak{u}_{-}^{(i)}
$$

Let $P_{k}^{+}$be the set of level $k$ integrable dominant $\mathfrak{g}$-weights, i.e.

$$
P_{k}^{+}=\left\{\lambda \in \mathfrak{h}^{*}: \lambda\left(\alpha_{i}^{\vee}\right) \in \mathbb{Z}_{\geq 0}, \lambda(K)=k\right\} .
$$

We also denote by $P_{k}^{\prime+} \hookrightarrow \mathfrak{h}^{\prime *}$ the image of $P_{k}^{+}$with respect to the projection $\mathfrak{h}^{*} \rightarrow \mathfrak{h}^{\prime *}, \lambda \mapsto \lambda^{\prime}$. For $\lambda \in P_{k}^{+}$let $L_{\lambda}$ be an integrable highest weight $\mathfrak{g}$ module with highest weight vector $v_{\lambda} \in L_{\lambda}$ such that

$$
\mathfrak{n} v_{\lambda}=0, \mathrm{U}\left(\mathfrak{n}_{-}\right) v_{\lambda}=L_{\lambda}, h\left(v_{\lambda}\right)=\lambda(h) v_{\lambda}, h \in \mathfrak{h} .
$$

Let $L_{\lambda^{\prime}}$ be $\mathfrak{g}^{\prime}$ module which coincides with $L_{\lambda}$ as a vector space and the action of $\mathfrak{g}^{\prime}$ is a restriction of the action of $\mathfrak{g}$. For any $\alpha \in \mathfrak{h}^{\prime *}$ set

$$
\left(L_{\lambda}\right)^{\alpha}=\left\{v \in L_{\lambda}: h v=\alpha(h) v \forall h \in \mathfrak{h}^{\prime}\right\} .
$$

Note that $L_{\lambda}$ is graded by an operator $d$. We set

$$
\operatorname{ch}_{q}\left(L_{\lambda}\right)^{\alpha}=\left.\operatorname{Tr} q^{d}\right|_{\left(L_{\lambda}\right)^{\alpha}} .
$$


Fix $\lambda_{1}^{\prime} \in P_{k_{1}}^{\prime+}, \lambda_{2}^{\prime} \in P_{k_{2}}^{\prime+}$, and consider the decomposition of the tensor product of $\mathfrak{g}^{\prime}$-modules:

$$
L_{\lambda_{1}^{\prime}} \otimes L_{\lambda_{2}^{\prime}}=\bigoplus_{\mu^{\prime} \in P_{k_{1}+k_{2}}^{\prime+}} C_{\lambda_{1}^{\prime} \lambda_{2}^{\prime}}^{\mu^{\prime}} \otimes L_{\mu^{\prime}} .
$$

The space $C_{\lambda_{1}^{\prime} \lambda_{2}^{\prime}}^{\mu^{\prime}}$ can be identified with a subspace of highest weight vectors of $\mathfrak{h}^{\prime}$-weight $\mu^{\prime}$ in $L_{\lambda_{1}^{\prime}} \otimes L_{\lambda_{2}^{\prime}}$. To define a character of $C_{\lambda_{1}^{\prime} \lambda_{2}^{\prime}}^{\mu^{\prime}}$ one needs to fix an action of the operator $d$ on each $\mathfrak{g}^{\prime}$ module $L_{\lambda^{\prime}}$. Note that if $\left.\lambda\right|_{\mathfrak{h}^{\prime}}=\left.\bar{\lambda}\right|_{\mathfrak{h}^{\prime}}$ then $L_{\lambda^{\prime}} \simeq L_{\bar{\lambda}^{\prime}}$. Therefore an action of $d$ on $L_{\lambda^{\prime}}$ depends on the choice of $\lambda(d)$. It is convenient for us to choose a normalization $\lambda_{1}(d)=\lambda_{2}(d)=\mu(d)=0$. This defines the characters of $L_{\lambda_{1}^{\prime}} \otimes L_{\lambda_{2}^{\prime}}$ and of $C_{\lambda_{1}^{\prime} \lambda_{2}^{\prime}}^{\mu^{\prime}}$. The character $\operatorname{ch}_{q} C_{\lambda_{1}^{\prime} \lambda_{2}^{\prime}}^{\mu^{\prime}}$ is called $\mathfrak{g}$ branching function and is denoted by $c_{\lambda_{1}^{\prime} \lambda_{2}^{\prime}}^{\mu^{\prime}}(q)$ :

$$
c_{\lambda_{1}^{\prime} \lambda_{2}^{\prime}}^{\mu^{\prime}}(q)=\left.\operatorname{Tr} q^{d}\right|_{C_{\lambda_{1}^{\prime} \lambda_{2}^{\prime}}^{\mu^{\prime}}}
$$

Recall that spaces $C_{\lambda_{1}^{\prime} \lambda_{2}^{\prime}}^{\mu^{\prime}}$ appear in the conformal field theory as spaces of states of coset theories (see [DMS]). Namely the Sugawara construction defines an action of the Virasoro algebra with generators $L_{n}$ on each $L_{\lambda^{\prime}}$. In particular for the operator $L_{0}$ one has

$$
L_{0} v_{\lambda}=\triangle_{\lambda^{\prime}} v_{\lambda},\left[L_{0}, x \otimes t^{i}\right]=-i x \otimes t^{i}
$$

( $\triangle_{\lambda^{\prime}}$ is a conformal weight). Now the GKO construction (see [GKO]) defines an action of Vir on the tensor product $L_{\lambda_{1}^{\prime}} \otimes L_{\lambda_{2}^{\prime}}$ which commutes with the diagonal action of $\mathfrak{g}^{\prime}$. Namely one puts

$$
L_{n}^{G K O}=L_{n}^{(1)} \otimes \mathrm{Id}+\mathrm{Id} \otimes L_{n}^{(2)}-L_{n}^{\text {diag }},
$$

where $L_{n}^{(1)}, L_{n}^{(2)}$ and $L_{n}^{\text {diag }}$ are Sugawara operators acting on $L_{\lambda_{1}^{\prime}}, L_{\lambda_{2}^{\prime}}$ and $L_{\lambda_{1}^{\prime}} \otimes L_{\lambda_{2}^{\prime}}$ respectively. Therefore, we obtain a structure of Vir-module on $C_{\lambda_{1}^{\prime} \lambda_{2}^{\prime}}^{\mu^{\prime}}$ and an equality

$$
\left.\operatorname{Tr} q^{L_{0}}\right|_{C_{\lambda_{1}^{\prime} \lambda_{2}^{\prime}}^{\mu^{\prime}}}=c_{\lambda_{1}^{\prime} \lambda_{2}^{\prime}}^{\mu^{\prime}}(q) q^{\triangle_{\lambda_{1}^{\prime}}+\triangle_{\lambda_{2}^{\prime}}-\triangle_{\mu^{\prime}}},
$$

where the left hand side is a character of the space of states of the corresponding coset model.

In the end of this section we recall the Weyl group notations, the GarlandLepowsky $\mathfrak{n}_{-}$-homology theorem and the BGG resolution. Let $W$ be the Weyl group of $\mathfrak{g}$, generated by simple reflections $s_{i}$. We denote by $l(w)$ the length of an element $w \in W$. Recall that the shifted action of $W$ on $\mathfrak{h}^{*}$ is given by $w * \lambda=w(\lambda+\rho)-\rho$, where $\rho\left(\alpha_{i}^{\vee}\right)=1$. We will need the following lemma:

Lemma 1.1. a) If $\lambda \in P_{k}^{+}$and $w * \lambda \in P_{k}^{+}$then $w=e$.

b) If $(w * \lambda) \alpha_{i}^{\vee} \leq-1$ for some $\lambda \in P_{k}^{+}$then $l\left(s_{i} w\right)<l(w)$. 
Proof. To prove $a)$ we rewrite an equality $w * \lambda=\lambda_{1}$ as $w(\lambda+\rho)=\lambda_{1}+\rho$. But if $\lambda, \lambda_{1} \in P_{k}^{+}$then

$$
(\lambda+\rho) \alpha_{i}^{\vee}>0, \quad\left(\lambda_{1}+\rho\right) \alpha_{i}^{\vee}>0 \quad \forall i .
$$

Therefore both $\lambda+\rho$ and $\lambda_{1}+\rho$ are the elements of the Weyl dominant chamber and so $\lambda=\lambda_{1}$. But from $w * \lambda=\lambda$ one gets $w=e$ (see Lemma 3.2.5 from Kum $)$.

We now prove $b)$. Note that $(w * \lambda) \alpha_{i}^{\vee} \leq-1$ is equivalent to

$$
(\lambda+\rho)\left(w^{-1} \alpha_{i}^{\vee}\right) \leq 0 .
$$

This gives $w^{-1} \alpha_{i}^{\vee}=\sum_{i=1}^{n} c_{j} \alpha_{j}^{\vee}$ with $c_{j} \leq 0$. Therefore $l\left(w^{-1} s_{i}\right)<l(w)$. Lemma is proved.

The following theorem is proved in GL:

Theorem. For any $\lambda \in P_{k}^{+}$we have an isomorphism of $\mathfrak{h}$-modules:

$$
H_{p}\left(\mathfrak{n}_{-}, L_{\lambda}\right) \simeq \bigoplus_{\substack{w \in W \\ l(w)=p}} \mathbb{C}_{w * \lambda},
$$

where $\mathbb{C}_{w * \lambda}$ is one-dimensional $\mathfrak{h}$-module of the weight $w * \lambda$.

We will also need the BGG resolution of integrable irreducible representations $L_{\lambda}$ (see BGG, Kum). Namely there exists an exact sequence of $\mathfrak{g}$-modules and $\mathfrak{g}$-homomorphism

$$
\ldots \rightarrow \bigoplus_{\substack{w \in W \\ l(w)=p}} M_{w * \lambda} \rightarrow \ldots \rightarrow M_{\lambda} \rightarrow L_{\lambda} \rightarrow 0,
$$

where $M_{\mu}$ is the weight $\mu$ Verma module.

\section{First homological BOSONiC FORMUla}

Lemma 2.1. For any $\lambda_{1} \in P_{k_{1}}^{+}, \lambda_{2} \in P_{k_{2}}^{+}$and $\mu \in P_{k_{1}+k_{2}}^{+}$we have:

$$
\begin{gathered}
C_{\lambda_{1}^{\prime} \lambda_{2}^{\prime}}^{\mu^{\prime}} \simeq H_{0}\left(\mathfrak{n}_{-}, L_{\lambda_{1}} \otimes L_{\lambda_{2}}\right)^{\mu^{\prime}}, \\
H_{p}\left(\mathfrak{n}_{-}, L_{\lambda_{1}} \otimes L_{\lambda_{2}}\right)^{\mu^{\prime}}=0 \text { for all } p>0 .
\end{gathered}
$$

Proof. Recall that for any $\lambda \in P_{k}^{+}$and $e \neq w \in W$ one has $w * \lambda \notin P_{k}^{+}$and so $(w * \lambda)^{\prime} \notin P_{k}^{\prime}+$. Therefore, from Garland-Lepowsky theorem we obtain that $H_{p}\left(\mathfrak{n}_{-}, L_{\lambda}\right)^{\mu}=0$ unless $p=0$ and $\lambda=\mu$. Now our lemma follows from the decomposition (10) and an equality

$$
H_{0}\left(\mathfrak{n}_{-}, L_{\lambda_{1}} \otimes L_{\lambda_{2}}\right)^{\mu^{\prime}} \simeq \bigoplus_{\substack{\left.\bar{\mu} \in P_{k}^{+} \\ \bar{\mu}\right|_{\mathfrak{h}^{\prime}}=\mu^{\prime}}} H_{0}\left(\mathfrak{n}_{-}, L_{\lambda_{1}} \otimes L_{\lambda_{2}}\right)^{\bar{\mu}} .
$$


Corollary 2.1. For any $\lambda_{1} \in P_{k_{1}}^{+}, \lambda_{2} \in P_{k_{2}}^{+}$and $\mu \in P_{k_{1}+k_{2}}^{+}$one has

$$
c_{\lambda_{1}^{\prime} \lambda_{2}^{\prime}}^{\mu^{\prime}}(q)=\sum_{p \geq 0}(-1)^{p} \operatorname{ch}_{q} H_{p}\left(\mathfrak{n}_{-}, L_{\lambda_{1}} \otimes L_{\lambda_{2}}\right)^{\mu^{\prime}} .
$$

We now compute the Euler characteristics (12) using the BGG-resolution of $L_{\lambda_{1}}$. Tensoring (11) by $L_{\lambda_{2}}$ we obtain the $\mathrm{U}\left(\mathfrak{n}_{-}\right)$-free resolution of $L_{\lambda_{1}} \otimes$ $L_{\lambda_{2}}$. Therefore the following complex counts $H_{p}\left(\mathfrak{n}_{-}, L_{\lambda_{1}} \otimes L_{\lambda_{2}}\right)$ :

$$
\ldots \rightarrow \mathbb{C} \otimes_{\mathrm{U}\left(\mathfrak{n}_{-}\right)}\left(L_{\lambda_{2}} \otimes F_{1}\right) \rightarrow \mathbb{C} \otimes_{\mathrm{U}\left(\mathfrak{n}_{-}\right)}\left(L_{\lambda_{2}} \otimes F_{0}\right) \rightarrow 0,
$$

where $F_{p}=\bigoplus_{l(w)=p} M_{w * \lambda_{1}}$. We can rewrite (13) as

$$
\ldots \rightarrow\left(\mathbb{C} \otimes_{\mathrm{U}\left(\mathfrak{n}_{-}\right)} F_{1}\right) \otimes L_{\lambda_{2}} \rightarrow\left(\mathbb{C} \otimes_{\mathrm{U}\left(\mathfrak{n}_{-}\right)} F_{0}\right) \otimes L_{\lambda_{2}} \rightarrow 0 .
$$

\section{Lemma 2.2.}

$$
\begin{aligned}
\sum_{p \geq 0}(-1)^{p} \operatorname{ch}_{q} H_{p}\left(\mathfrak{n}_{-}, L_{\lambda_{1}} \otimes L_{\lambda_{2}}\right)^{\mu^{\prime}} & = \\
& \sum_{p \geq 0}(-1)^{p} \sum_{l(w)=p} q^{\left(w * \lambda_{1}\right) d} \operatorname{ch}_{q}\left(L_{\lambda_{2}}\right)^{\left(\mu-w * \lambda_{1}\right)^{\prime}} .
\end{aligned}
$$

Proof. Recall that $F_{p}=\bigoplus_{l(w)=p} M_{w * \lambda_{1}}$. Therefore,

$$
\mathbb{C} \otimes_{\mathrm{U}\left(\mathfrak{n}_{-}\right)} F_{p}=\bigoplus_{l(w)=p} \mathbb{C}_{w * \lambda_{1}}
$$

Now our lemma follows from the equality of Euler characteristics of the complex (14) and the right hand side of (12).

Proposition 2.1. We have a bosonic formula for the branching functions:

$$
c_{\lambda_{1}^{\prime} \lambda_{2}^{\prime}}^{\mu^{\prime}}(q)=\sum_{p \geq 0}(-1)^{p} \sum_{\substack{w \in W \\ l(w)=p}} q^{\left(w * \lambda_{1}\right) d} \operatorname{ch}_{q}\left(L_{\lambda_{2}}\right)^{\left(\mu-w * \lambda_{1}\right)^{\prime}}
$$

Proof. Follows from Corollary 2.1 and Lemma 2.2

Remark 2.1. We can use the $B G G$ resolution of $L_{\lambda_{2}}$ instead of $L_{\lambda_{1}}$. This interchanges $\lambda_{1}$ and $\lambda_{2}$ in the right hand side of (15) and leads to another formula for branching functions $c_{\lambda_{1}^{\prime} \lambda_{2}^{\prime}}^{\mu^{\prime}}(q)$.

\section{SECOND HOMOLOGICAL BOSONIC FORMUlA}

In this section we study homology $H_{p}\left(\mathfrak{n}_{-}, L_{\mu} \otimes L_{\lambda_{1}}^{*}\right)$ replacing the tensor product $L_{\lambda_{1}} \otimes L_{\lambda_{2}}$ from the previous section by the "fraction" $L_{\mu} \otimes L_{\lambda_{1}}^{*}$. We note that though $L_{\mu} \otimes L_{\lambda_{1}}^{*}$ does not belong to the category $\mathcal{O}$ (the eigenvalues of the operator $d$ are not bounded from below) it is still integrable. So we first prove some statements about integrable representations.

Recall the definition (9) of the subalgebra $\mathfrak{u}_{-}^{(i)}$. 
Lemma 3.1. Let $M$ be an integrable $\mathfrak{g}$ module. Then

$$
H_{n}\left(\mathfrak{n}_{-}, M\right) \simeq H_{0}\left(\mathfrak{g}_{-\alpha_{i}}, H_{n}\left(\mathfrak{u}_{-}^{(i)}, M\right)\right) \oplus H_{1}\left(\mathfrak{g}_{-\alpha_{i}}, H_{n-1}\left(\mathfrak{u}_{-}^{(i)}, M\right)\right) .
$$

Proof. We consider the Hochschild-Serre spectral sequence associated with a pair $\mathfrak{u}_{-}^{(i)} \hookrightarrow \mathfrak{n}_{-}$. Note that $\mathfrak{u}_{-}^{(i)}$ is an ideal and $\mathfrak{n}_{-} / \mathfrak{u}_{-}^{(i)} \simeq \mathfrak{g}_{-\alpha_{i}}$. The second term of this spectral sequence is given by

$$
E_{p, q}^{2}=H_{p}\left(\mathfrak{g}_{-\alpha_{i}}, H_{q}\left(\mathfrak{u}_{-}^{(i)}, M\right)\right) .
$$

We prove our lemma by showing that $E_{p, q}^{2}=E_{p, q}^{\infty}$.

Because of the integrability condition $M$ is a direct sum of irreducible finite-dimensional $\mathfrak{s l}_{2}^{(i)}$ modules. Therefore the same is true for $\Lambda^{q}\left(\mathfrak{u}_{-}^{(i)}\right) \otimes M$ and also for $H_{q}\left(\mathfrak{u}_{-}^{(i)}, M\right)$. For any nonnegative integer $s$ we denote by $\pi_{s}$ an irreducible $\mathfrak{s l}_{2}$ module with highest weight $s\left(\operatorname{dim} \pi_{s}=s+1\right)$ and fix highest and lowest weight vectors $v_{s}$ and $u_{s}$. Let $\pi_{s} \hookrightarrow H_{q}\left(\mathfrak{u}_{-}^{(i)}, M\right)$ be a direct summand and

$$
\alpha_{p} \in \Lambda^{p}\left(\mathfrak{g}_{-\alpha_{i}}\right) \otimes \pi_{s}
$$

be a chain representing some class in $H_{p}\left(\mathfrak{g}_{-\alpha_{i}}, \pi_{s}\right) \hookrightarrow H_{p}\left(\mathfrak{g}_{-\alpha_{i}}, H_{q}\left(\mathfrak{u}_{-}^{(i)}, M\right)\right)$. We set

$$
\alpha_{0}=v_{s}, \quad \alpha_{1}=f_{i} \otimes u_{s} .
$$

Let $\beta_{p} \in \Lambda^{p}\left(\mathfrak{g}_{-\alpha_{i}}\right) \otimes \Lambda^{q}\left(\mathfrak{u}_{-}^{(i)}\right) \otimes M$ be the chains of the form

$$
\beta_{0}=x_{0}, \quad \beta_{1}=f_{i} \otimes x_{1}
$$

which represent $\alpha_{p}$ (i.e. $x_{0}$ represents $v_{s}$ and $x_{1}$ represents $u_{s}$ ). Now let $d_{\mathfrak{n}_{-}}\left(d_{\mathfrak{u}_{-}^{(i)}}\right)$ be the differential in the standard complex for $H_{n}\left(\mathfrak{n}_{-}, M\right)$ $\left(H_{n}\left(\mathfrak{u}_{-}^{(i)}, M\right)\right)$. We state that $d_{\mathfrak{n}_{-}} \beta_{p}=0$. In fact, for $p=0$ this just follows from $v_{s} \in H_{q}\left(\mathfrak{u}_{-}^{(i)}, M\right)$. Now let $p=1$. Then

$$
d_{\mathfrak{n}_{-}} \beta_{1}=d_{\mathfrak{n}_{-}}\left(f_{i} \otimes x_{1}\right) .
$$

We know that $d_{\mathfrak{u}_{-}^{(i)}} x_{1}=0$ and $f_{i} x_{1}=0$ (because $x_{1}$ represents the lowest weight vector). This gives $d_{\mathfrak{n}_{-}} \beta_{1}=0$. But because of $d_{\mathfrak{n}_{-}} \beta=0$ we obtain that differentials $d_{2}, d_{3}, \ldots$ in the Hochschild-Serre spectral sequence are trivial and $E_{p, q}^{2}=E_{p, q}^{\infty}$. Lemma is proved.

Corollary 3.1. Let $M$ be an integrable level $k \mathfrak{g}$ module. Then

$$
H_{0}\left(\mathfrak{n}_{-}, M\right)^{\lambda}=0 \text { unless } \lambda \in P_{k}^{+} .
$$

Proof. Because of Lemma 3.1 we obtain

$$
H_{0}\left(\mathfrak{n}_{-}, M\right) \simeq H_{0}\left(\mathfrak{g}_{-\alpha_{i}}, H_{0}\left(\mathfrak{u}_{-}^{(i)}, M\right)\right)
$$

for all $i=1, \ldots, n$. We recall that $H_{0}\left(\mathfrak{g}_{-\alpha_{i}}, \pi_{s}\right)$ is one-dimensional space of the $\alpha_{i}^{\vee}$-weight $s$. Therefore, because $H_{0}\left(\mathfrak{u}_{-}^{(i)}, M\right)$ is a direct sum of finite-dimensional $\mathfrak{s i}_{2}^{(i)}$ modules, we obtain $\lambda\left(\alpha_{i}^{\vee}\right) \in \mathbb{Z}_{\geq 0}$ for any weight $\lambda$ of $H_{0}(\mathfrak{n}, M)$. This gives $\lambda \in P_{k}^{+}$. 
Proposition 3.1. Let $M$ be an integrable level $k \mathfrak{g}$ module. Then

a) $H_{n}\left(\mathfrak{n}_{-}, M\right)^{w * \lambda}=0$ for $w \in W, \lambda \in P_{k}^{+}$if $l(w)>n$.

b) $H_{n}\left(\mathfrak{n}_{-}, M\right)^{w * \lambda} \simeq H_{n-l(w)}\left(\mathfrak{n}_{-}, M\right)^{\lambda}$ for any $w \in W, \alpha \in P_{k}^{+}$such that $l(w) \leq n$.

Proof. We prove $a$ ) and $b$ ) simultaneously using

$$
H_{n}\left(\mathfrak{n}_{-}, M\right)^{\mu} \simeq H_{0}\left(\mathfrak{g}_{-\alpha_{i}}, H_{n}\left(\mathfrak{u}_{-}^{(i)}, M\right)\right)^{\mu} \oplus H_{1}\left(\mathfrak{g}_{-\alpha_{i}}, H_{n-1}\left(\mathfrak{u}_{-}^{(i)}, M\right)\right)^{\mu}
$$

and the induction on $n$. The case $n=0$ follows from Corollary 3.1. Suppose our lemma is proved for $m<n$. We assume $l(w)>0$ (otherwise $a$ ) and $b$ ) are trivial). Then there exists $i$ such that

$$
(w * \lambda) \alpha_{i}^{\vee} \leq-1 \text {. }
$$

We have

$$
H_{n}\left(\mathfrak{n}_{-}, M\right)^{w * \lambda} \simeq H_{0}\left(\mathfrak{g}_{-\alpha_{i}}, H_{n}\left(\mathfrak{u}_{-}^{(i)}, M\right)\right)^{w * \lambda} \oplus H_{1}\left(\mathfrak{g}_{-\alpha_{i}}, H_{n-1}\left(\mathfrak{u}_{-}^{(i)}, M\right)\right)^{w * \lambda} .
$$

Because of the condition (16) we have

$$
H_{0}\left(\mathfrak{g}_{-\alpha_{i}}, H_{n}\left(\mathfrak{u}_{-}^{(i)}, M\right)\right)^{w * \lambda}=0
$$

and therefore

$$
\begin{aligned}
H_{n}(\mathfrak{n}, M)^{w * \lambda} \simeq H_{1}\left(\mathfrak{g}_{-\alpha_{i}}, H_{n-1}\left(\mathfrak{u}_{-}^{(i)}, M\right)\right)^{w * \lambda} & \simeq \\
& H_{0}\left(\mathfrak{g}_{-\alpha_{i}}, H_{n-1}\left(\mathfrak{u}_{-}^{(i)}, M\right)\right)^{\left(s_{i} w\right) * \lambda},
\end{aligned}
$$

because

$$
H_{1}\left(\mathfrak{g}_{-\alpha_{i}}, \pi_{s}\right)^{\alpha} \simeq H_{0}\left(\mathfrak{g}_{-\alpha_{i}}, \pi_{s}\right)^{s_{i} * \alpha}
$$

for any $\alpha$. We also know that

$$
\begin{aligned}
H_{n-1}\left(\mathfrak{n}_{-}, M\right)^{\left(s_{i} w\right) * \lambda} \simeq H_{0}\left(\mathfrak{g}_{-\alpha_{i}}, H_{n-1}\left(\mathfrak{u}_{-}^{(i)}, M\right)\right)^{\left(s_{i} w\right) * \lambda} \oplus & \\
& H_{1}\left(\mathfrak{g}_{-\alpha_{i}}, H_{n-2}\left(\mathfrak{u}_{-}^{(i)}, M\right)\right)^{\left(s_{i} w\right) * \lambda}
\end{aligned}
$$

and because of $\left(\left(s_{i} w\right) * \lambda\right) \alpha_{i}^{\vee}=-(w * \lambda) \alpha_{i}^{\vee}-2 \geq-1$ (see (16)) $)$ we obtain

$$
H_{1}\left(\mathfrak{g}_{-\alpha_{i}}, H_{n-2}(\mathfrak{u}, M)\right)^{\left(s_{i} w\right) * \lambda}=0
$$

(because $H_{1}\left(\mathfrak{g}_{-\alpha_{i}}, \pi_{s}\right)^{t}=0$ for any $t \geq-1$ ). Therefore

$$
H_{n-1}\left(\mathfrak{n}_{-}, M\right)^{\left(s_{i} w\right) * \lambda} \simeq H_{0}\left(\mathfrak{g}_{-\alpha_{i}}, H_{n-1}\left(\mathfrak{u}_{-}^{(i)}, M\right)\right)^{\left(s_{i} w\right) * \lambda} .
$$

From (17) and (18) we obtain

$$
H_{n}\left(\mathfrak{n}_{-}, M\right)^{w * \lambda} \simeq H_{n-1}\left(\mathfrak{n}_{-}, M\right)^{\left(s_{i} w\right) * \lambda}
$$

Note that because of (16) and Lemma 1.1 $l\left(s_{i} w\right)=l(w)-1$.

Now suppose that $n<l(w)$. Then iterating (19) we obtain

$$
H_{n}\left(\mathfrak{n}_{-}, M\right)^{w * \lambda} \simeq H_{0}\left(\mathfrak{n}_{-}, M\right)^{w^{\prime} * \lambda}
$$

for some $w^{\prime}$ with $l\left(w^{\prime}\right)>0$. But this homology vanishes because of Corollary 3.1. This gives $a$ ). To obtain $b$ ) one needs to iterate (19). Proposition is proved. 
Let $\omega: \mathfrak{g} \rightarrow \mathfrak{g}$ be the Chevalley involution defined by $e_{i} \rightarrow-f_{i}, f_{i} \rightarrow-e_{i}$, $h \rightarrow-h(h \in \mathfrak{h})$. For $\mathfrak{g}$ module $V$ we denote by $V^{\omega}$ a $\mathfrak{g}$ module which coincides with $V$ as a vector space and the action of $\mathfrak{g}$ is twisted by $\omega$.

Lemma 3.2. Let $M$ be some $\mathfrak{g}$ module, $\lambda \in \mathfrak{h}^{*}$. Then

$$
H_{n}\left(\mathfrak{n}_{-}, M\right)^{\lambda} \simeq H_{n}\left(\mathfrak{g}, \mathfrak{h}, M \otimes M_{\lambda}^{\omega}\right),
$$

where $M_{\lambda}$ is the Verma module.

Proof. We first rewrite

$$
H_{n}\left(\mathfrak{n}_{-}, M\right)^{\lambda} \simeq H_{n}\left(\mathfrak{b}_{-}, \mathfrak{h}, M \otimes \mathbb{C}_{-\lambda}\right),
$$

where $\mathbb{C}_{-\lambda}$ is one-dimensional $\mathfrak{b}_{-}=\mathfrak{n}_{-} \oplus \mathfrak{h}$-module with trivial action of $\mathfrak{n}_{-}$. Now our lemma follows from

$$
\operatorname{Ind}_{\mathfrak{b}_{-}}^{\mathfrak{g}}\left(M \otimes \mathbb{C}_{-\lambda}\right) \simeq M \otimes M_{\lambda}^{\omega}
$$

In what follows we study homology $H_{n}\left(\mathfrak{n}_{-}, L_{\mu} \otimes L_{\lambda_{1}}^{*}\right)^{\lambda_{2}}$ for the triple of weights $\lambda_{1} \in P_{k_{1}}^{+}, \lambda_{2} \in P_{k_{2}}^{+}, \mu \in P_{k_{1}+k_{2}}^{+}$. Note that $L_{\mu} \otimes L_{\lambda_{1}}^{*}$ is integrable $\mathfrak{g}$ module. Because of Lemma 3.2 we have an isomorphism

$$
H_{n}\left(\mathfrak{n}_{-}, L_{\mu} \otimes L_{\lambda_{1}}^{*}\right)^{\lambda_{2}} \simeq H_{n}\left(\mathfrak{g}, \mathfrak{h}, L_{\mu} \otimes L_{\lambda_{1}}^{*} \otimes M_{\lambda_{2}}^{\omega}\right)
$$

Proposition 3.2. There exists a spectral sequence $\bar{E}_{p, q}^{r}$ with

$$
\bar{E}_{p, q}^{1}=\bigoplus_{w: l(w)=p} H_{q}\left(\mathfrak{n}_{-}, L_{\mu} \otimes L_{\lambda_{1}}^{*}\right)^{w * \lambda_{2}}
$$

such that $\bar{E}_{p, q}^{r}$ converges to $H_{\bullet}\left(\mathfrak{g}, \mathfrak{h}, L_{\mu} \otimes L_{\lambda_{1}}^{*} \otimes L_{\lambda_{2}}^{*}\right)$. In addition $\bar{E}_{p, q}^{1}=0$ for $p>q$.

Proof. We first note that $\bar{E}_{p, q}^{1}=0$ for $p>q$ because of part $a$ ) of Proposition 3.1.

Now consider the BGG resolution

$$
\ldots \rightarrow F_{\lambda}(2) \rightarrow F_{\lambda}(1) \rightarrow F_{\lambda}(0) \rightarrow L_{\lambda} \rightarrow 0, F_{\lambda}(p)=\bigoplus_{w: l(w)=p} M_{w * \lambda} .
$$

Recall that for any $\lambda \in P_{k}^{+}\left(L_{\lambda}^{*}\right)^{\omega} \simeq L_{\lambda}$. We thus obtain the dual BGGresolution

$$
\ldots \rightarrow F_{\lambda}^{\omega}(2) \rightarrow F_{\lambda}^{\omega}(1) \rightarrow F_{\lambda}^{\omega}(0) \rightarrow L_{\lambda}^{*} \rightarrow 0, F_{\lambda}^{\omega}(p)=\bigoplus_{w: l(w)=p} M_{w * \lambda}^{\omega} .
$$

This gives the following resolution

$$
(20) \ldots \rightarrow L_{\mu} \otimes L_{\lambda_{1}}^{*} \otimes F_{\lambda_{2}}^{\omega}(1) \rightarrow L_{\mu} \otimes L_{\lambda_{1}}^{*} \otimes F_{\lambda_{2}}^{\omega}(0) \rightarrow L_{\mu} \otimes L_{\lambda_{1}}^{*} \otimes L_{\lambda_{2}}^{*} \rightarrow 0 .
$$

In order to establish a connection between

$$
H_{q}\left(\mathfrak{g}, \mathfrak{h}, L_{\mu} \otimes L_{\lambda_{1}}^{*} \otimes L_{\lambda_{2}}^{*}\right) \text { and } H_{q}\left(\mathfrak{n}_{-}, L_{\mu} \otimes L_{\lambda_{1}}^{*}\right)^{w * \lambda_{2}}
$$


we use a bi-complex $K_{p, q}$ associated with the resolution (20):

$$
K_{p, q}=\left[\Lambda^{q}(\mathfrak{g} / \mathfrak{h}) \otimes L_{\mu} \otimes L_{\lambda_{1}}^{*} \otimes F_{\lambda_{2}}^{\omega}(p)\right]^{0}
$$

(the space of $\mathfrak{h}$-invariants). The first term of the corresponding spectral sequence is given by

$$
\bar{E}_{p, q}^{1}=H_{q}\left(\mathfrak{g}, \mathfrak{h}, L_{\mu} \otimes L_{\lambda_{1}}^{*} \otimes F_{\lambda_{2}}^{\omega}(p)\right)
$$

and $\bar{E}_{p, q}^{r}$ converges to $H_{n}\left(\mathfrak{g}, \mathfrak{h}, L_{\mu} \otimes L_{\lambda_{1}}^{*} \otimes L_{\lambda_{2}}^{*}\right)$. From the definition of $F_{\lambda_{2}}^{\omega}(p)$ and Lemma 3.2 we obtain

$$
\bar{E}_{p, q}^{1}=\bigoplus_{w: l(w)=p} H_{q}\left(\mathfrak{n}_{-}, L_{\mu} \otimes L_{\lambda_{1}}^{*}\right)^{w * \lambda_{2}}
$$

Proposition is proved.

Corollary 3.2. $H_{0}\left(\mathfrak{n}_{-}, L_{\mu} \otimes L_{\lambda_{1}}^{*}\right)^{\lambda_{2}^{\prime}} \simeq\left(C_{\lambda_{1}^{\prime} \lambda_{2}^{\prime}}^{\mu^{\prime}}\right)^{*}$.

Proof. Note that $H_{0}\left(\mathfrak{n}_{-}, L_{\mu} \otimes L_{\lambda_{1}}^{*}\right)^{\lambda_{2}^{\prime}}$ is isomorphic to

$$
\bigoplus_{\substack{\left.\bar{\lambda}_{2} \in P_{k}^{+} \\ \bar{\lambda}_{2}\right|_{\mathfrak{h}^{\prime}}=\lambda_{2}^{\prime}}} H_{0}\left(\mathfrak{n}_{-}, L_{\mu} \otimes L_{\lambda_{1}}^{*}\right)^{\bar{\lambda}_{2}}
$$

Fix some $\bar{\lambda}_{2}$ with $\left.\bar{\lambda}_{2}\right|_{\mathfrak{h}^{\prime}}=\lambda_{2}^{\prime}$. Then using the spectral sequence from the Proposition 3.2 we obtain

$$
H_{0}\left(\mathfrak{n}_{-}, L_{\mu} \otimes L_{\lambda_{1}}^{*}\right)^{\bar{\lambda}_{2}}=\bar{E}_{0,0}^{1}
$$

and $\bar{E}_{n, 0}^{1}=0$ for all $n>0$. Therefore $\lim _{r \rightarrow \infty} \bar{E}_{0,0}^{r}=\bar{E}_{0,0}^{1}$. In addition

$$
\bar{E}_{0,0}^{\infty} \simeq H_{0}\left(\mathfrak{g}, \mathfrak{h}, L_{\mu} \otimes L_{\lambda_{1}}^{*} \otimes L_{\bar{\lambda}_{2}}^{*}\right) \simeq H^{0}\left(\mathfrak{g}, \mathfrak{h}, L_{\mu}^{*} \otimes L_{\lambda_{1}} \otimes L_{\bar{\lambda}_{2}}\right)^{*} .
$$

Now our Corollary follows from

$$
H^{0}\left(\mathfrak{g}, \mathfrak{h}, L_{\mu}^{*} \otimes L_{\lambda_{1}} \otimes L_{\bar{\lambda}_{2}}\right)^{*} \simeq \operatorname{Hom}_{\mathfrak{g}}\left(L_{\mu}, L_{\lambda_{1}} \otimes L_{\bar{\lambda}_{2}}\right)^{*}
$$

and

$$
\bigoplus_{\substack{\left.\bar{\lambda}_{2} \in P_{k}^{+} \\ \bar{\lambda}_{2}\right|_{\mathfrak{h}^{\prime}}=\lambda_{2}^{\prime}}} H^{0}\left(\mathfrak{g}, \mathfrak{h}, L_{\mu}^{*} \otimes L_{\lambda_{1}} \otimes L_{\bar{\lambda}_{2}}\right)^{*} \simeq \operatorname{Hom}_{\mathfrak{g}^{\prime}}\left(L_{\mu^{\prime}}, L_{\lambda_{1}^{\prime}} \otimes L_{\bar{\lambda}_{2}^{\prime}}\right)^{*}
$$

We now study the special case of $H_{n}\left(\mathfrak{n}_{-}, L_{\mu} \otimes L_{\lambda_{1}}^{*}\right)^{\lambda_{2}}$ with $\lambda_{2}=0$ and $\mu=\lambda_{1}$.

Lemma 3.3. $H_{n}\left(\mathfrak{n}_{-}, L_{\mu} \otimes L_{\mu}^{*}\right)^{0}=0$ for $n>0$.

Proof. We consider a filtration $\left(L_{\mu}^{*}\right)_{m}$ on $L_{\mu}^{*}$ :

$$
\left(L_{\mu}^{*}\right)_{m}=\operatorname{span}\left\langle e_{i_{1}} \ldots e_{i_{s}} v_{\mu}^{*}, s \leq m, 1 \leq i_{l} \leq n\right\rangle,
$$

where $v_{\mu}^{*}$ is a lowest weight vector of $L_{\mu}^{*}$. This induces a filtration

$$
\left(\left[\Lambda^{n}\left(\mathfrak{n}_{-}\right) \otimes L_{\mu} \otimes L_{\mu}^{*}\right]^{0}\right)_{m}=\left[\Lambda^{n}\left(\mathfrak{n}_{-}\right) \otimes L_{\mu} \otimes\left(L_{\mu}^{*}\right)_{m}\right]^{0} .
$$


For the associated spectral sequence one has

$$
E_{n, m}^{1}=\left[H_{n+m}\left(\mathfrak{n}_{-}, L_{\mu}\right) \otimes\left(L_{\mu}^{*}\right)_{m} /\left(L_{\mu}^{*}\right)_{m-1}\right]^{0} .
$$

Because of $H_{n+m}\left(\mathfrak{n}_{-}, L_{\mu}\right) \simeq \bigoplus_{w: l(w)=n+m} \mathbb{C}_{w * \mu}$ we obtain

$$
E_{n, m}^{1}=\bigoplus_{w: l(w)=n+m}\left[\left(L_{\mu}^{*}\right)_{m} /\left(L_{\mu}^{*}\right)_{m-1}\right]^{-w * \mu}
$$

But

$$
\left(L_{\mu}^{*}\right)^{-w * \mu} \simeq\left(L_{\mu}^{*}\right)^{-w \mu+\rho-w \rho} \simeq\left(L_{\mu}^{*}\right)^{-\mu+w^{-1} \rho-\rho}=0
$$

because $w^{-1} \rho-\rho<0$ for $l(w)>0$. This gives $E_{n, m}^{1}=0$ for $n+m \neq 0$. Lemma is proved.

In the following Lemma we calculate homology $H_{n}\left(\mathfrak{g}, \mathfrak{h}, L_{\mu} \otimes L_{\nu}^{*}\right)$ for two weights $\mu, \nu \in P_{k_{1}+k_{2}}^{+}$.

Lemma 3.4. Let $\mu, \nu \in P_{k_{1}+k_{2}}^{+}$. Then

$$
\operatorname{dim} H_{2 n}\left(\mathfrak{g}, \mathfrak{h}, L_{\mu} \otimes L_{\nu}^{*}\right)=\delta_{\mu, \nu} \#\{w \in W: l(w)=n\} .
$$

In addition $H_{2 n-1}\left(\mathfrak{g}, \mathfrak{h}, L_{\mu} \otimes L_{\nu}^{*}\right)=0$.

Proof. Because of the isomorphism $\left(L_{\nu}^{*}\right)^{\omega} \simeq L_{\nu}$ the BGG resolution gives the following resolution:

$$
\ldots \rightarrow \bigoplus_{\substack{w_{1}, w_{2} \in W \\ l\left(w_{1}\right)+l\left(w_{2}\right)=p}} M_{w_{1} * \mu} \otimes M_{w_{2} * \nu}^{\omega} \rightarrow \ldots \rightarrow M_{\mu} \otimes M_{\nu}^{\omega} \rightarrow L_{\mu} \otimes L_{\nu}^{*} \rightarrow 0 .
$$

This resolution is $(\mathfrak{g}, \mathfrak{h})$-free. Therefore a complex $G_{\bullet}$ with

$$
G_{p}=\bigoplus_{\substack{w_{1}, w_{2} \in W \\ l\left(w_{1}\right)+l\left(w_{2}\right)=p}}\left[\mathbb{C} \otimes_{U(\mathfrak{g} / \mathfrak{h})}\left(M_{w_{1} * \mu} \otimes M_{w_{2} * \nu}^{\omega}\right)\right]^{0}
$$

counts $H_{p}\left(\mathfrak{g}, \mathfrak{h}, L_{\mu} \otimes L_{\nu}^{*}\right)$. Note that $G_{p}=\bigoplus_{\substack{l\left(w_{1}\right)+l\left(w_{2}\right)=p \\ w_{1} * \mu=w_{2} * \nu}} \mathbb{C}_{0}$. In view of $\mu, \nu \in P_{k_{1}+k_{2}}^{+}$the condition $w_{1} * \mu=w_{2} * \nu$ is equivalent to $w_{1}=w_{2}, \nu=\mu$. Therefore $G_{2 n-1}=0, n \geq 1$ and $\operatorname{dim} G_{2 n}=\delta_{\mu \nu} \#\{w \in W: l(w)=n\}$. Lemma is proved.

Proposition 3.3. The natural map

$$
\tau: H_{n}\left(\mathfrak{g}, \mathfrak{h}, L_{\mu} \otimes L_{\lambda_{1}}^{*} \otimes M_{\lambda_{2}}^{\omega}\right) \rightarrow H_{n}\left(\mathfrak{g}, \mathfrak{h}, L_{\mu} \otimes L_{\lambda_{1}}^{*} \otimes L_{\lambda_{2}}^{*}\right)
$$

is trivial for $n>0$.

Proof. Because of an isomorphism

$$
H_{n}\left(\mathfrak{g}, \mathfrak{h}, L_{\mu} \otimes L_{\lambda_{1}}^{*} \otimes M_{\lambda_{2}}^{\omega}\right) \simeq H_{n}\left(\mathfrak{b}_{-}, \mathfrak{h}, L_{\mu} \otimes L_{\lambda_{1}}^{*} \otimes \mathbb{C}_{\lambda_{2}}^{*}\right)
$$

it is enough to prove that the map

$$
\tau^{\prime}: H_{n}\left(\mathfrak{b}_{-}, \mathfrak{h}, L_{\mu} \otimes L_{\lambda_{1}}^{*} \otimes \mathbb{C}_{\lambda_{2}}^{*}\right) \rightarrow H_{n}\left(\mathfrak{g}, \mathfrak{h}, L_{\mu} \otimes L_{\lambda_{1}}^{*} \otimes L_{\lambda_{2}}^{*}\right)
$$


is trivial. Note that $\tau^{\prime}$ comes from the natural embedding

(21) $\quad \imath:\left[\Lambda^{n}\left(\mathfrak{b}_{-} / \mathfrak{h}\right) \otimes L_{\mu} \otimes L_{\lambda_{1}}^{*} \otimes \mathbb{C}_{\lambda_{2}}^{*}\right]^{0} \hookrightarrow\left[\Lambda^{n}(\mathfrak{g} / \mathfrak{h}) \otimes L_{\mu} \otimes L_{\lambda_{1}}^{*} \otimes L_{\lambda_{2}}^{*}\right]^{0}$.

Therefore it suffices to show that any chain

$$
c \in\left[\Lambda^{n}\left(\mathfrak{b}_{-} / \mathfrak{h}\right) \otimes L_{\mu} \otimes L_{\lambda_{1}}^{*} \otimes L_{\lambda_{2}}^{*}\right]^{0} \hookrightarrow\left[\Lambda^{n}(\mathfrak{g} / \mathfrak{h}) \otimes L_{\mu} \otimes L_{\lambda_{1}}^{*} \otimes L_{\lambda_{2}}^{*}\right]^{0}
$$

defines a trivial class in $H_{n}\left(\mathfrak{g}, \mathfrak{h}, L_{\mu} \otimes L_{\lambda_{1}}^{*} \otimes L_{\lambda_{2}}^{*}\right)$.

Because of Lemma 3.4 it is enough to show that any chain from

$$
\left[\Lambda^{n}\left(\mathfrak{n}_{-}\right) \otimes L_{\mu} \otimes L_{\mu}^{*}\right]^{0} \hookrightarrow\left[\Lambda^{n}(\mathfrak{g} / \mathfrak{h}) \otimes L_{\mu} \otimes L_{\lambda_{1}}^{*} \otimes L_{\lambda_{2}}^{*}\right]^{0}
$$

defines a trivial element in $H_{n}\left(\mathfrak{g}, \mathfrak{h}, L_{\mu} \otimes L_{\lambda_{1}}^{*} \otimes L_{\lambda_{2}}^{*}\right)$ for $n>0$. But from Lemma 3.3 we know that

$$
H_{n}\left(\mathfrak{n}_{-}, L_{\mu} \otimes L_{\mu}^{*}\right)^{0}=0 \text { for } n>0
$$

Proposition is proved.

Theorem 3.1. For any $\lambda_{1} \in P_{k_{1}}^{+}, \lambda_{2} \in P_{k_{2}}^{+}, \mu \in P_{k_{1}+k_{2}}^{+}$and $n>0$

$$
H_{n}\left(\mathfrak{n}_{-}, L_{\mu} \otimes L_{\lambda_{1}}^{*}\right)^{\lambda_{2}}=0
$$

Proof. We use the spectral sequence from Proposition 3.2 .

$$
\bar{E}_{p, q}^{1}=\bigoplus_{w: l(w)=p} H_{q}\left(\mathfrak{n}_{-}, L_{\mu} \otimes L_{\lambda_{1}}^{*}\right)^{w * \lambda_{2}} \simeq \bigoplus_{l(w)=p} H_{q}\left(\mathfrak{g}, \mathfrak{h}, L_{\mu} \otimes L_{\lambda_{1}}^{*} \otimes M_{w * \lambda_{2}}^{\omega}\right) .
$$

From Corollary 3.2 we obtain $\bar{E}_{0,0}^{1} \simeq\left(C_{\lambda_{1}^{\prime} \lambda_{2}^{\prime}}^{\mu^{\prime}}\right)^{*}$ and therefore

$$
\bar{E}_{k, k}^{1} \simeq \bigoplus_{w: l(w)=k}\left(C_{\lambda_{1}^{\prime} \lambda_{2}^{\prime}}^{\mu^{\prime}}\right)^{*}
$$

In addition Propositions 3.1 and 3.2 gives

$$
\bar{E}_{p, n+p}^{1} \simeq \bar{E}_{0, n}^{1}, \bar{E}_{p, q}^{1}=0 \text { for } p>q .
$$

Our goal is to show that $\bar{E}_{0, n}^{1}=0, n>0$. (Because of (23) this is equivalent to the proof of $\bar{E}_{p, q}^{1}=0$ for $p \neq q$ ). Note that this agrees with a fact that $\bar{E}_{k, k}^{1}$ is isomorphic to $H_{2 k}\left(\mathfrak{g}, \mathfrak{h}, L_{\mu} \otimes L_{\lambda_{1}}^{*} \otimes L_{\lambda_{1}}^{*}\right.$ ) (see Lemma 3.4 and (22)).

We prove the statement $\bar{E}_{0,2 n-1}^{1}=0, \bar{E}_{0,2 n}^{1}=0$ by induction on $n \geq 1$. First let $n=1$. Because of Proposition 3.3 the map

$$
\bar{E}_{0,2}^{1} \rightarrow H_{2}\left(\mathfrak{g}, \mathfrak{h}, L_{\mu} \otimes L_{\lambda_{1}}^{*} \otimes L_{\lambda_{1}}^{*}\right)
$$

is trivial and therefore $\bar{E}_{0,2}^{\infty}=0$. This gives $\bar{E}_{1,1}^{\infty}=\bar{E}_{1,1}^{1}$, because

$$
\bar{E}_{1,1}^{1} \simeq H_{2}\left(\mathfrak{g}, \mathfrak{h}, L_{\mu} \otimes L_{\lambda_{1}}^{*} \otimes L_{\lambda_{1}}^{*}\right) \simeq \bar{E}_{1,1}^{\infty} \oplus \bar{E}_{2,0}^{\infty} .
$$

So a differential $d_{1}: \bar{E}_{1,1}^{1} \rightarrow \bar{E}_{0,1}^{1}$ is trivial and

$$
\bar{E}_{0,1}^{1}=\bar{E}_{0,1}^{\infty}=H_{1}\left(\mathfrak{g}, \mathfrak{h}, L_{\mu} \otimes L_{\lambda_{1}}^{*} \otimes L_{\lambda_{1}}^{*}\right)=0 .
$$

According to Proposition 3.1 we obtain $\bar{E}_{1,2}^{1}=0$, which gives

$$
\bar{E}_{0,2}^{1}=\bar{E}_{0,2}^{\infty}=0 \text {. }
$$


Now suppose $\bar{E}_{0, s}^{1}=0$ for $s \leq 2(n-1)$. This gives $\bar{E}_{p, s+p}^{1}=0$ for $s \leq 2(n-1), p \geq 0$. Note that the map

$$
\bar{E}_{0,2 n}^{1} \rightarrow H_{2 n}\left(\mathfrak{g}, \mathfrak{h}, L_{\mu} \otimes L_{\lambda_{1}}^{*} \otimes L_{\lambda_{1}}^{*}\right)
$$

is trivial and so $\bar{E}_{0,2 n}^{\infty}=0$. Recall that the differential $d_{r}$ acts from $\bar{E}_{p, q}^{r}$ to $\bar{E}_{p-r, q+r-1}^{r}$. This gives

$$
\bar{E}_{p, q}^{r}=\bar{E}_{p, q}^{1} \text { for } p+q \leq 2 n .
$$

Because of

$$
H_{2 n}\left(\mathfrak{g}, \mathfrak{h}, L_{\mu} \otimes L_{\lambda_{1}}^{*} \otimes L_{\lambda_{1}}^{*}\right) \simeq \bar{E}_{n, n}^{1}
$$

we have $\bar{E}_{n, n}^{1}=\bar{E}_{n, n}^{\infty}$. So the differential $d_{n}: \bar{E}_{n, n}^{n} \rightarrow \bar{E}_{0,2 n-1}^{n}$ is trivial. Therefore

$$
0=\bar{E}_{0,2 n-1}^{\infty}=\bar{E}_{0,2 n-1}^{n}=\bar{E}_{0,2 n-1}^{1}
$$

$\left(\bar{E}_{0,2 n-1}^{\infty}=0\right.$ because by induction assumption we know that $\bar{E}_{p, q}^{\infty}=0$ for $p+q=2 n-1,(p, q) \neq(2 n-1,0))$. The equality $\bar{E}_{0,2 n-1}^{1}=0$ gives $\bar{E}_{1,2 n}^{1}=0$. Therefore,

Theorem is proved.

$$
0=\bar{E}_{0,2 n}^{\infty}=\bar{E}_{0,2 n}^{1} .
$$

Corollary 3.3. For any $\lambda_{1} \in P_{k_{1}}^{+}, \lambda_{2} \in P_{k_{2}}^{+}, \mu \in P_{k_{1}+k_{2}}^{+}$and $n>0$

$$
H_{n}\left(\mathfrak{n}_{-}, L_{\mu} \otimes L_{\lambda_{1}}^{*}\right)^{\lambda_{2}^{\prime}}=0
$$

Theorem 3.2. Let $\lambda_{1} \in P_{k_{1}}^{+}, \lambda_{2} \in P_{k_{2}}^{+}, \mu \in P_{k_{1}+k_{2}}^{+}$. Then

$$
c_{\lambda_{1}^{\prime} \lambda_{2}^{\prime}}^{\mu^{\prime}}\left(q^{-1}\right)=\sum_{p \geq 0}(-1)^{p} \sum_{\substack{w \in W \\ l(w)=p}} q^{(w * \mu) d} \operatorname{ch}_{q^{-1}}\left(L_{\lambda_{1}}\right)^{\left(w * \mu-\lambda_{2}\right)^{\prime}} .
$$

Another expressions for branching functions can be obtained by interchanging $\lambda_{1}$ and $\lambda_{2}$ in the above expression.

Proof. Consider homology $H_{n}\left(\mathfrak{n}_{-}, L_{\mu} \otimes L_{\lambda_{1}}^{*}\right)^{\lambda_{2}^{\prime}}$. Because of Corollary 3.2 and Theorem 3.1 we know that

$$
\sum_{p \geq 0}(-1)^{p} \operatorname{ch}_{q} H_{n}\left(\mathfrak{n}_{-}, L_{\mu} \otimes L_{\lambda_{1}}^{*}\right)^{\lambda_{2}^{\prime}}=c_{\lambda_{1}^{\prime} \lambda_{2}^{\prime}}^{\mu^{\prime}}\left(q^{-1}\right) .
$$

Using the BGG-resolution of $L_{\mu}$ we obtain that the following complex counts $H_{n}\left(\mathfrak{n}_{-}, L_{\mu} \otimes L_{\lambda_{1}}^{*}\right)^{\lambda_{2}^{\prime}}$ :

$$
\ldots \rightarrow D_{2} \rightarrow D_{1} \rightarrow D_{0} \rightarrow 0, D_{p}=\bigoplus_{w: l(w)=p}\left(\mathbb{C} \otimes_{\mathrm{U}\left(\mathfrak{n}_{-}\right)}\left(M_{w * \mu} \otimes L_{\lambda_{1}}^{*}\right)\right)^{\lambda_{2}^{\prime}} .
$$

We note that

$$
\left(\mathbb{C} \otimes_{\mathrm{U}\left(\mathfrak{n}_{-}\right)}\left(M_{w * \mu} \otimes L_{\lambda_{1}}^{*}\right)\right)^{\lambda_{2}^{\prime}} \simeq\left(\mathbb{C}_{w * \mu} \otimes L_{\lambda_{1}}^{*}\right)^{\lambda_{2}^{\prime}}
$$

and so we have

$$
\operatorname{ch}_{q}\left(\mathbb{C} \otimes_{\mathrm{U}\left(\mathfrak{n}_{-}\right)}\left(M_{w * \mu} \otimes L_{\lambda_{1}}^{*}\right)\right)^{\lambda_{2}^{\prime}}=q^{(w * \mu) d} \operatorname{ch}_{q}\left(L_{\lambda_{1}}^{*}\right)^{\left(-w * \mu+\lambda_{2}\right)^{\prime}} .
$$


Therefore the Euler characteristics of the complex (25) is given by

$$
\sum_{p \geq 0}(-1)^{p} \sum_{w: l(w)=p} q^{(w * \mu) d} \operatorname{ch}_{q^{-1}}\left(L_{\lambda_{1}}\right)^{\left(w * \mu-\lambda_{2}\right)^{\prime}} .
$$

But the Euler characteristics of (25) coincides with the sum

$$
\sum_{n \geq 0}(-1)^{n} \operatorname{ch}_{q} H_{n}\left(\mathfrak{n}_{-}, L_{\mu} \otimes L_{\lambda_{1}}^{*}\right)^{\lambda_{2}^{\prime}}
$$

Theorem is proved.

\section{THE $\widehat{\mathfrak{s l}_{2}}$ CASE}

In this section we specialize formulas (15) and (24) to the case $\mathfrak{g}=\widehat{\mathfrak{s l}_{2}}$.

Let $h$ be the standard generator of the Cartan subalgebra of $\mathfrak{s l}_{2}$. Then $\mathfrak{h}$ is spanned by $h_{0}=h \otimes 1, K$ and $d$. Define $(i, k, m) \in \mathfrak{h}^{*}$ by

$$
(i, k, m) h_{0}=i,(i, k, m) K=k,(i, k, m) d=m .
$$

Let $(i, k)=\left.(i, k, m)\right|_{\mathfrak{h}^{\prime}}$. Note that

$$
P_{k}^{\prime+}=\left\{(i, k) \in \mathfrak{h}^{\prime *}: i, k \in \mathbb{Z}_{\geq 0}, i \leq k\right\} .
$$

We denote by $L_{i, k}(0 \leq i \leq k)$ the highest weight irreducible representation of $\widehat{\mathfrak{s l}}_{2}^{\prime}$ with highest weight $(i, k)$. For fixed levels $k_{1}, k_{2}$ let $c_{i_{1} i_{2}}^{j}(q)$ be the corresponding branching functions defined as characters of $C_{i_{1} i_{2}}^{j}$ :

$$
L_{i_{1}, k_{2}} \otimes L_{i_{2}, k_{2}}=\bigoplus_{j=0}^{k_{1}+k_{2}} C_{i_{1} i_{2}}^{j} \otimes L_{j, k_{1}+k_{2}}
$$

Recall that for any $s>0$ there exist two elements $w_{s, 1}, w_{s, 2} \in W$ with $l\left(w_{s, i}\right)=s$. In addition

$$
\begin{gathered}
w_{2 n, 1} *(i, k, m)=(i+2 n(k+2), k, m+n(n(k+2)+i+1)), \\
w_{2 n, 2} *(i, k, m)=(i-2 n(k+2), k, m+n(n(k+2)-i-1)), \\
w_{2 n-1,1} *(i, k, m)=(-i-2+2 n(k+2), k, m+n(n(k+2)-i-1)), \\
w_{2 n+1,2} *(i, k, m)=(-i-2-2 n(k+2), k, m+n(n(k+2)+i+1)) .
\end{gathered}
$$

Let $V^{l}$ be the eigenspace of the operator $h \in \mathfrak{s l}_{2}$ with an eigenvalue $l$.

\section{Proposition 4.1.}

$$
c_{i_{1} i_{2}}^{j}(q)=\sum_{p \in \mathbb{Z}} q^{p^{2}\left(k_{1}+2\right)+p\left(i_{1}+1\right)}\left(\operatorname{ch}_{q} L_{i_{2}, k_{2}}^{2 p\left(k_{1}+2\right)-j+i_{1}}-\operatorname{ch}_{q} L_{i_{2}, k_{2}}^{2 p\left(k_{1}+2\right)+j+i_{1}+2}\right) .
$$

Proof. Follows from (15) and (26). 


\section{Proposition 4.2.}

$$
\begin{aligned}
& \text { (28) } c_{i_{1} i_{2}}^{j}(q)= \\
& \sum_{p \in \mathbb{Z}} q^{-\left(k_{1}+k_{2}+2\right) p^{2}-(j+1) p}\left(\operatorname{ch}_{q} L_{i_{1}, k_{1}}^{2\left(k_{1}+k_{2}+2\right) p+j-i_{2}}-\operatorname{ch}_{q} L_{i_{1}, k_{1}}^{2\left(k_{1}+k_{2}+2\right) p+j+i_{2}+2}\right) .
\end{aligned}
$$

Proof. Follows from (24) and (26).

Remark 4.1. Note that formula (28) coincides with bosonic formula from [F].

\section{REFERENCES}

[BNY] J. Bagger, D. Nemeschansky, S. Yankielowicz, Virasoro algebras with central charge $c>1$, Phys. Rev. Lett. 60 (1988), no. 5, 389-392.

[BGG] I.N. Bernstein, I.M. Gel'fand, S.I. Gel'fand, Differential operators on the base affine space and a study of $\mathfrak{g}$-modules, in: Lie groups and their representations (I.M. Gelfand ed.), Summer school of the Bolyai Janos Math. Soc., Halsted Press, 1975, 21-64.

[DJKMO] E. Date, M. Jimbo, A. Kuniba, T. Miwa, M. Okado, Exactly solvable SOS models: local heights probabilities and theta function identities, Nucl. Phys. B 290 (1987), no. 2, 231-273.

E. Date, M. Jimbo, A. Kuniba, T. Miwa, M. Okado, Exactly solvable SOS models I: proof of star-triangle relation and combinatorial identities, Adv. Stud. in Pure Math. 16 (1988), 17-122.

[DMS] P. Di Francesco, P. Mathieu and D. Sénéchal, Conformal field theory, Springer GTCP, New York, 1997.

[F] E. Feigin, Infinite fusion products and $\widehat{\mathfrak{s l}_{2}}$ cosets, preprint 2006, http://xxx.lanl.gov/abs/math.QA/0603226

[FOW] O. Foda, M. Okado, O. Warnaar, A proof of polynomial identities of type $\widehat{\mathfrak{s l}(n)_{1}} \otimes$ $\widehat{\mathfrak{s l}(n)_{1}} / \widehat{\mathfrak{s l}(n)_{2}}$, J. Math. Phys. 37 (1996), 965-986.

[FF] B. Feigin, E. Feigin, Homological realization of restricted Kostka polynomials, Int. Math. Res. Not. 2005, no. 33, pp. 1997-2029.

[FFJMT] B. Feigin, E. Feigin, M. Jimbo, T. Miwa, Y. Takeyama, A $\phi_{1,3}$-filtration for Virasoro minimal series $M\left(p, p^{\prime}\right)$ with $1<p^{\prime} / p<2$, preprint 2006, http://xxx.lanl.gov/abs/math.QA/0603070

[GKO] P. Goddard, A. Kent, D. Olive, Unitary representations of the Virasoro and superVirasoro algebras, Comm. Math. Phys., 103 (1986), no.1, 105-119.

[GL] H. Garland, J. Lepowsky, Lie algebra homology and the Macdonald-Kac formulas, Invent. Math. 34 (1976), 37-76.

[Kac] V. Kac, Infinite dimensional Lie algebras, 3rd ed., Cambridge University Press, Cambridge, 1990.

[KMQ] D. Kastor, E. Martinec, Z. Qiu, Current algebra and conformal decrete series, Phys. Lett. B200 (1988), no. 4, 434-440.

[Kum] S. Kumar, Kac-Moody groups, their flag varieties and representation theory, Progressin Mathematics, vol. 204, Birkhauser Boston, Massachusetts, 2002.

[R] F. Ravanini, An infinite class of new conformal field theories with extended algebras, Mod. Phys. Lett. A3 (1988), no. 4, 397-412.

[RC] A. Rocha-Caridi, Vacuum vector representations of the Virasoro algebra, Vertex Operators in Mathematical Physics (Berkeley, Calif., 1983), Math. Sci. Res. Inst. Publ., vol. 3, Springer, New York, 1985, pp. 451-473. 
[S1] A. Schilling, Multinomials and polynomial bosonic forms for the branching functions of the $\widehat{\mathfrak{s u}}(2)_{M} \times \widehat{\mathfrak{s u}}(2)_{N} / \widehat{\mathfrak{s u}}(2)_{M+N}$ conformal coset models, Nucl. Phys. B467 (1996), $247-271$.

[S2] A. Schilling, Polynomial fermionic forms for the branching functions of the rational coset conformal field theories $\widehat{\mathfrak{s u}}(2)_{M} \times \widehat{\mathfrak{s u}}(2)_{N} / \widehat{\mathfrak{s u}}(2)_{M+N}$, Nucl. Phys. B459 (1996), 393-436.

[SS] A. Schilling, M. Shimozono, Fermionic formulas for level-restricted generalized Kostka polynomials and coset branching functions, Comm. Math. Phys. 220 (2001), no. 1, 105-164.

Evgeny FEIGIN:

Tamm Theory Division, Lebedev Physics Institute, Russian Academy of Sciences,

Russia, 119991, Moscow, Leninski prospect, 53

AND

Independent University of Moscow,

Russia, Moscow, 119002, Bol'shoi Vlas'evskii, 11

E-mail address: evgfeig@mccme.ru 